\title{
Woman With Polyuria And Polydipsia
}

\author{
Jeffrey Clough, MSIV, and Andrew Rose, MD
}

\section{Case Presentation}

A 20 year-old Caucasian female with a past medical history significant only for occasional headaches and anemia presents with a chief complaint of increasing thirst for two weeks. The patient was in her usual state of health until approximately two weeks prior to admission when she noted the gradual onset of increasing thirst and began drinking more and more water over the next few days. Her thirst increased to the point where she drank an estimated 18 liters of fluid during the 24 hours prior to admission. This thirst was accompanied by frequent urination, which would often awaken her during the night and she noted her urine to be very clear. These symptoms began within a few days of a vaginal yeast infection, for which she was treated with one dose of fluconazole. She denied any other symptoms during this episode.

Her past medical history was significant for occasional non-focal headaches without aura or specific precipitants. These headaches had been present for several years and responded well to NSAIDS. She also reports that she has a history of anemia and occasionally takes iron supplements. She does not report taking regular medications and has never had surgery. Her last menstrual period was approximately three weeks earlier. She is a college student, works part-time in a pharmacy and lives with her family and claims to be happy with her life. She is in a monogamous relationship with her boyfriend and reports no problems. She denies alcohol, cigarette, or illicit substance use. Her family history is significant only for hypertension, diabetes mellitus, and stroke on her maternal side and gastric cancer in her maternal grandfather. She denies any neurologic, psychiatric, or autoimmune disease in the family.

On physical examination, the patient appeared comfortable, wellhydrated, and her vital signs were normal. Her examination revealed no abnormalities, with the exception of hippus of the pupils. Full neurologic examination was normal including visual field testing with confrontational visual fields. Initial laboratory examination revealed a mild microcytic anemia with a hemoglobin of $11.9 \mathrm{~g} / \mathrm{dL}$ and a normal chemistry panel including a serum sodium of $139 \mathrm{meq} / \mathrm{dL}$ and creatinine of $0.5 \mathrm{mg} / \mathrm{dL}$.

\section{Differential Diagnosis and Evaluation}

The most likely diagnoses given this patient's history are diabetes insipidus (DI) or psychogenic polydipsia. DI can be further classified into nephrogenic or central. Nephrogenic DI is less likely in this case as it generally occurs either as a genetic disease presenting early in life in males or as a side effect of medications such as lithium or demeclocyline. It may also be secondary to hypercalcemia, which was normal on her laboratory evaluation. Therefore, central DI and psychogenic polydipsia were the remaining likely diagnoses. Both of these disorders are common to the patient's demographic. Central DI is caused by destruction of the vasopressin producing cells in the posterior pituitary gland. It is most often an idiopathic phenomenon likely to be autoimmune, but other causes include infiltrative disorders such as hemochro- matosis and sarcoidosis, as well as pituitary neoplasms. Psychogenic polydipsia is also idiopathic and is thought to be caused by dysfunction of thirst-regulating nuclei in the hypothalamus. It is often associated with patients who have a psychiatric disorder but can occur with infiltrative disorders and in otherwise normal patients. Psychogenic polydipsia differs from DI in that patients are driven to drink water and they urinate to compensate for the increased volume load whereas in DI patients cannot concentrate their urine and must drink to replace the lost fluids.

The primary method to distinguish between these three disorders is the water deprivation test. In this test, the patient remains NPO and urine osmolarity is measured every hour. Additionally, the serum osmolarity and sodium level are measured every 2 hours. The test is stopped if the patient's urine is concentrated to a level of $600 \mathrm{mOsm}$ in which case DI is ruled out. If the serum osmolarity exceeds $295 \mathrm{mOsm}$ or the urine osmolarity is stable for 2-3 consecutive hours (less than $10 \%$ increase) while the serum osmolarity is rising, DI is the likely diagnosis. At this point, 5 $10 \mathrm{mcg}$ of vasopressin is injected subcutaneously and the urine osmolarity is measured one hour later. If the urine osmolarity increases by $50 \%$ or more, the patient is considered to have central DI. In many cases of central DI, the disease is only partial and can be diagnosed if the urine osmolarity increases by $15-50 \% .{ }^{1}$ If the patient has nephrogenic DI, the urine osmolarity should not significantly increase.

In addition to water deprivation test, MRI of the brain should be obtained to rule out any obvious causes of central DI such as a mass. In idiopathic central DI, the characteristic finding on MRI is loss of the pituitary bright spot and thickening of the pituitary stalk. If central DI is determined, a pituitary panel should be obtained to rule out any other pituitary dysfunction.

The results of the water deprivation test in this patient are shown in Table 1. The patient was unable to concentrate her urine greater than $250 \mathrm{mOsm}$ at 6 hours even though her serum osmolarity rose to $297 \mathrm{mOsm}$ after 4 hours. This indicated that she had diabetes insipidus rather than psychogenic polydipsia. She was given $5 \mathrm{mcg}$ of subcutaneous vasopressin and her urine osmolarity rose to $349 \mathrm{mOsm}$. Although this did not fulfill the $50 \%$ increase requirement, it did surpass the $15 \%$ requirement for partial central DI. With the high pretest likelihood of central DI rather than nephrogenic DI, it was felt that she had partial central DI. Interestingly, the patient reported a complete resolution of her symptoms of thirst following administration of vasopressin. In addition, MRI revealed loss of the posterior hypophyseal bright spot and thickening of the pituitary stalk consistent with central DI. She was started on $10 \mathrm{mcg}$ of nasal desmopressin twice daily and discharged the next day with instructions to obtain outpatient blood work to monitor her electrolytes in one week and follow up with the endocrinologist in 2 weeks. A pituitary panel was drawn during the hospital stay with the results to be followed by her endocrinologist. 
Table 1. Water deprivation test

\begin{tabular}{|c|c|c|c|c|c|c|c|c|}
\hline Time & $8: 00$ & $9: 00$ & $10: 00$ & $11: 00$ & $12: 00$ & $13: 00$ & $14: 00^{*}$ & $15: 00$ \\
\hline $\mathrm{Na}(\mathrm{mmol} / \mathrm{L})$ & 142 & & 141 & & & & & \\
\hline Serum osmolarity (mOsm) & & & 294 & & 297 & & & \\
\hline \multicolumn{9}{|l|}{ Urine osmolarity } \\
\hline$(\mathrm{mOsm})$ & 105 & 202 & 288 & 269 & & & 250 & 349 \\
\hline
\end{tabular}

* $5 \mathrm{mcg}$ vasopressin administered at this time.

\section{Discussion}

Idiopathic DI accounts for $30-50 \%$ of cases of central DI. ${ }^{2}$ Autoimmunity is thought to be an important cause of idiopathic central DI. Pivonello et al. found that patients with idiopathic central DI were significantly more likely than control patients to display antibodies to arginine vasopressin-secreting cells. Age less than 30, known autoimmune disease, and radiological evidence of pituitary stalk thickening were all independent risk factors for having a positive antibody assay. A patient with idiopathic DI and all of these criteria has a $99 \%$ chance of having these antibodies. ${ }^{3}$ This patient had two of these parameters, which placed her at an $80 \%$ chance of having these autoimmune antibodies. While there is no definitive proof that these antibodies are the cause of central $\mathrm{DI}$, this is a plausible explanation given our understanding of autoimmunity.
To our knowledge, there are no known case reports of an association between vaginal yeast infections or fluconazole and central diabetes insipidus. The coincidental timing and likely autoimmunity of this patient's disease may suggest either the yeast infection or fluconazole treatment as a possible trigger for the disease. Although this represents only one case of central DI following a vaginal yeast infection with fluconazole treatment, further such reports might allow researchers to study and understand this interesting disease.

\section{References:}

1. Zerbe RL; Robertson GL A comparison of plasma vasopressin measurements with a standard indirect test in the differential diagnosis of polyuria. N Engl J Med 1981 Dec 24;305(26):1539-46.

2. Makaryus AN. McFarlane SI. Diabetes insipidus: diagnosis and treatment of a complex disease. Cleveland Clinic Journal of Medicine. 73(1):65-71, 2006 Jan

3. Pivonello R, De Bellis A, Faggiano A, Di Salle F, Petretta M, Di Somma C, Perrino S, Altucci P, Bizzarro A, Bellastella A, Lombardi G, Colao A. Central diabetes insipidus and autoimmunity: relationship between the occurrence of antibodies to arginine vasopressin-secreting cells and clinical, immunological, and radiological features in a large cohort of patients with central diabetes insipidus of known and unknown etiology.

4. J Clin Endocrinol Metab. 2003 Apr;88(4):1629-36. 17th Centery Pinjore

Reflecting Pool,

Pinjore, India

Photo courtesy of

Vaibhav Menendiratta, MD

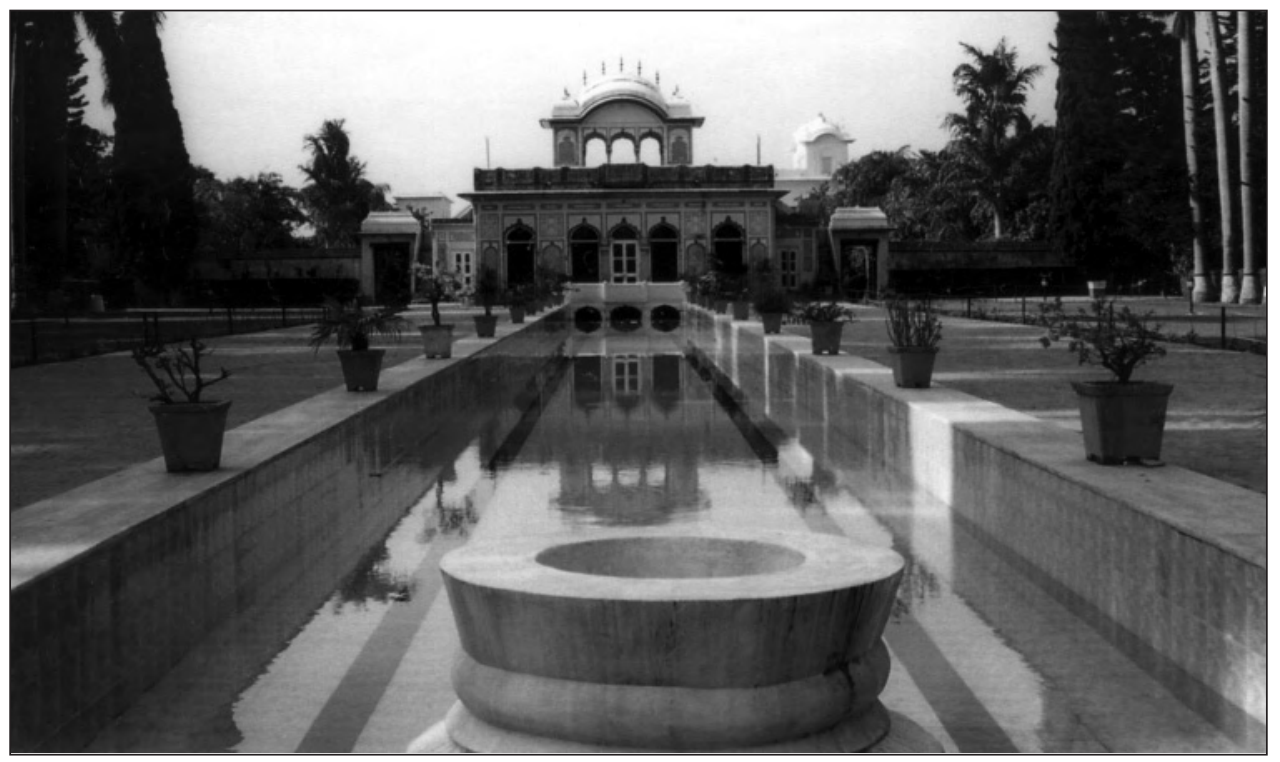

